\title{
Are Dental Medical Services Shared Equitably between the Members of our Society, Regarding Their Treatment Needs?
}

\author{
Petrescu Bianca Nausica ${ }^{1}$, Bondor Cosmina' ${ }^{2}$ Boșca Adina Bianca ${ }^{3}$, Câmpian Radu Septimiu' ${ }^{1}$, Buhățel Dan ${ }^{1}$, \\ Husti Ioan Liviư ${ }^{4}$ Băbțan Anida Maria ${ }^{1}$, Crișan Horațiu Traian ${ }^{*}$ and Ilea Aranka ${ }^{1}$ \\ ${ }^{1}$ Department of Oral Rehabilitation, Oral Health and Dental Office Management, Faculty of Dentistry, "Iuliu Haţieganu" University of Medicine and Pharmacy \\ Cluj-Napoca, Romania \\ ${ }^{2}$ Department of Biomedical Informatics and Biostatistics, Faculty of Medicine, "Iuliu Haţieganu" University of Medicine and Pharmacy Cluj-Napoca, Romania \\ ${ }^{3}$ Department of Histology, Faculty of Medicine, "Iuliu Haţieganu" University of Medicine and Pharmacy Cluj-Napoca, Romania \\ ${ }^{4}$ Radiodent, Cluj-Napoca, Romania
}

${ }^{5}$ Department of Medical Education, Abilities-Human Sciences, Faculty of Medicine, "Iuliu Haţieganu" University of Medicine and Pharmacy Cluj-Napoca, Romania

*Corresponding author: Crișan Horaţiu Traian, PhD, Department of Medical Education, Abilities-Human Sciences, Faculty of Medicine, "Iuliu Haţieganu" University of Medicine and Pharmacy Cluj-Napoca, Gheorghe Marinescu, No 23, Cluj-Napoca, Romania, E-mail: “horatiu.crisan@umfcluj.ro"horatiu.crisan@umfcluj.ro.

Received Date: March 14, 2019

Published Date: March 29, 2019

\begin{abstract}
Health is a required element for a balanced human existence. The patients' inability to pay for medical services limits their access to medical care and aggravates a disease which could be cured earlier with fewer resources. Beyond the impact of dental pathology on the individual through pain and discomfort, with subsequent consequences on general health and quality of life, negative effects are reflected on the entire community, affecting the healthcare system well as the economic system.
\end{abstract}

Keywords: Dental medical services; Oral health; Health care; Medical insurance

\section{Introduction}

In today's Romania, taking the year 2015 as a reference, more than $99.7 \%$ of independent dental practices are private, of which only $27.31 \%$ have a contract with the National Health Insurance House in order to provide oro-dental services for insured persons through the national health insurance system [1]. Although in Romania, all companies are obliged to contribute to the health care budget, by paying for the basic public medical insurance of their employees, who in turn also pay, regardless of their types of revenue, state medical insurance including oro-dental care. The latest public information (National Health Insurance House Financial Report, 2015) showed that the average expenditure for oro-dental care in 2015 was 4.6455 ron (1.0289€) per insured person [2]. Even though according to the law, each health insured person has an equal right to benefit from certain oro-dental care services, this general constitutional and legal right to health care is obviously limited not only by the total health budget, but also by the much reduced expenditure in the field of oro-dental care, which in 2015 was $0.3625 \%$ of the total amount spent on health care.

Under the circumstances of very limited insurance fund allocations, regardless of the patients' oro-dental needs, their financial capacity to cover the expenses of their own treatment should be taken into account.

This article is meant to raise a number of issues to be debated:

a. Do patients with low income have proper access to dental treatments, through the national insurance system?

b. Is the human right to health respected and fulfilled?

c. What do we suggest as an achievable solution for this community problem?

d. Do health care programs help improve the overall dental status and quality of life of patients for whom they are intended? 
If the patients don't have the financial support to prevent the dental pathologies or to go to the dentist early in the pathology evolution, the delay of the treatment will have severe consequences on the oral cavity health and on the overall health of the patient [36]. The low income of our country is more generalized, compared to other countries, but the other countries also have this problem.

One of the main differences among Romania and other countries such as the U.S.A. regarding access to oro-dental services is the fact that all state insured adults have access, despite the limited budget, to dental services, whereas in the U.S.A., only in few states, adults can have access to these services under Medicaid. Even if children with Medical insurance had more visits to the dentists between 2000 and 2013, there was a decline among adults with private insurance over the same timeframe (American Dental Association).

Similar with the situation in Romania, in the U.S.A. there is a discrepancy between the access to oro-dental services for different groups of patients, based on the type of their health insurance, whereas in Romania it is based upon ability to pay directly for private oro-dental services. It is probable that the reasons of the romanian patients of not going to the dentist would be similar to those in the U.S.A., where the main reason given by those who did not visit the dentist in the last 12 months was the cost of the dental services, regardless of income, age, or source of dental benefits factors (American Dental Association).

The lack of financial resources leads to fewer dental checkups and fewer dental problems treated in their early stages. This determines higher cost treatments, which cannot be covered by dental insurances.

This kind of data is needed to determine the amount of resources and dental professionals required for treating this category of population and consequently, for improving the quality of life and the productivity of the entire community [7-10].

A study published in Norway regarding institutionalized mentally disabled persons over 15 years of age [11]. As the age of the person increases, the number of present teeth decreases [12]. In Romania, there are four main health care funding forms: the national health care system, social health insurance, private health insurance, and direct payment. Private health insurance is optional and is provided by companies other than the government. The national health care system, which is managed by the government does not have enough money to cover all dental services. Dental services are fully covered only for children, widows and persons with disabilities. All other persons have to pay for any dental services, to private dental care providers, entirely or partially (if the private dental care provider has a contract with the National Health Insurance House). Many of the patients who cannot afford to pay directly the dental medical services to the private dental care providers, do not have access to dental health care. Therefore, people who address to the private dental services are people who had medium to high income (in 2010 medium income was 334.11€; in 2015 medium income was $415.8 €$ - National Institute of Statistics - Monthly earnings - 1991-2017).
Many literature sources consider periodontal disease to be one of the major causes of tooth loss [13-18]. Other previous studies on institutionalized elderly people in Western Australia ( $56 \%$ of all dentate patients), as well as a study on 60 to 80 -year-old Swedish patients $(35 \%)$, showed high proportions of patients with teeth affected by periodontal disease $[19,20]$. There are many studies that highlight the severity of dental disease in the case of low-income patients [21-23]. From a legal point of view, all patients have an equal right to publicly financed oro-dental treatment. From a moral perspective, people belonging to disadvantaged groups, regardless of their being health insured or not, based on their lack of ability to pay for any kind of treatment, should be included in foreground public oro-dental treatment programs. Briefly expressed, our thesis is that the members of disadvantaged groups should be prioritized in being granted dental treatment through public oral health programs.

It is obvious that our argument implies advocacy of the necessity to augment the amount of resources spent on oro-dental care, allocated from the Romanian health care budget. The fact that these resources are today so limited involves a deep cleavage among potential oro-dental patients. More precisely, it entails a cleavage between those who afford dental treatments at their own expense and those who do not have the necessary financial resources for oro-dental treatments. The latter usually belong to disadvantaged groups and are implicitly excluded from any oro-dental care, unless granted by public health treatment.

\section{Conclusion}

In order to prevent a decline in these persons' level of wellbeing up to a critical threshold, a few circumstantial arguments based on empirical research would suffice to ground a moral right of disadvantaged group members to get priority access to publicly funded treatment. Moreover, at least from a normative point of view, public resources allocated for oro-dental treatment should increase up to a sufficient level for the members of disadvantaged groups to benefit from a similar treatment to that of patients who can afford private treatments.

\section{Acknowledgments}

This work was supported by the Doctoral Research Project (PCD 2016) of "Iuliu Haţieganu" University of Medicine and Pharmacy Cluj-Napoca - Contract no. 7690/15.04.2016 and by the COFUND-ERA-HDHL ERANET Project, European and International Cooperation - Subprogram 3.2 - Horizon 2020, PNCDI III Program - Biomarkers for Nutrition and Health - "Innovative technological approaches for validation of salivary AGEs as novel biomarkers in evaluation of risk factors in diet-related diseases", grant no 25/1.09.2017.

\section{Conflict of Interest}

The authors declare no conflict of interest.

\section{References}

1. (2015) The National Institute of Statistics.

2. (2015) The National Health Insurance House Financial Report. 
3. Mattila KJ, Valtonen VV, Nieminen M, Huttunen JK (1995) Dental infection and the risk of new coronary events: prospective study of patients with documented coronary artery disease. Clin Infect Dis 20(3): 588-592.

4. Joshipura KJ, Rimm EB, Douglass CW, Trichopoulos D, Ascherio A, et al. (1996) Poor oral health and coronary heart disease. J Dent Res 75(9): 1631-1636.

5. Wu T, Trevisan M, Genco RJ, Dorn JP, Falkner KL, et al. (2000) Periodonta disease and risk of cerebrovascular disease the first national health and nutrition examination survey and its follow-up study. Arch Intern Med 160(18): 2749-2755

6. Syrjänen J, Peltola J, Valtonen V, Iivanainen M, Kaste M, et al. (1989) Dental infections in association with cerebral infarction in young and middle-aged men. J Intern Med 225(3): 179-184.

7. Gordon M, Kusner W, Shifman A, Ronen E, Newburn E, et al. (1986) Assessing the dental treatment needs of an adult Israeli military population. Community Dent Oral Epidemiol 14: 244-249.

8. Bouma J, Van de Poel F (1985) Caries and total extraction in a mediumsized city in the Netherlands. Community Dent Oral Epidemiol 13(3): 168-172.

9. Mariño R, Giacaman RA (2014) Factors related to unmet oral health needs in older adults living in Chile. Arch Gerontol Geriatr 58(3): 454 459.

10. La Torre G, Romeo U, Iarocci G, Orlando Brugnoletti, Leda Semyonov, et al. (2015) Socio-demographic inequalities and teeth extraction in the last 12 months in Italy. Ann Stomatol 5(4): 131-135.

11. Svatun B, Helöe LA (1975) Dental status and treatment needs among institutionalized mentally subnormal persons. Community Dent Oral Epidemiol 3(5): 208-213.

12. Copeland LB, Krall EA, Brown LJ, Garcia RI, Streckfus CF, et al. (2004) Predictors of tooth loss in two US adult populations. J Public Health Dent 64(1): 31-37.
13. Morita M, Kimura T, Kanegae M, Ishikawa A, Watanabe T, et al. (1994) Reasons for extraction of permanent teeth in Japan. Community Dent Oral Epidemiol 22(5 Pt 1): 303-306.

14. Ong G, Yeo JF, Bhole S (1996) A survey of reasons for extraction of permanent teeth in Singapore. Community Dent Oral Epidemiol 24(2): 124-127.

15. Lo EC, Corbet EF, Holmgren CJ (1994) Oral health care needs among the middle-aged and the elderly in Hong Kong. Community Dent Oral Epidemiol 22(5 Pt 2): 403-407.

16. Reich E, Hiller KA (1993) Reasons for tooth extraction in the western states of Germany. Community Dent Oral Epidemiol 21: 379-383.

17. Murray H, Clarke M, Locker D, Kay EJ (1997) Reasons for tooth extractions in dental practices in Ontario, Canada according to tooth type. Int Dent J 47(1): 3-8.

18. Aida J, Ando Y, Akhter R, Aoyama H, Masui M, et al. (2006) Reasons for permanent tooth extractions in Japan. J Epidemiol 16(5): 214-219.

19. Stockwell AJ (1987) Survey of the oral health needs of institutionalized elderly patients in Western Australia. Community Dent Oral Epidemiol 15(5): 273-276

20. Fure S, Zickert I (1997) Incidence of tooth loss and dental caries in 60-, 70- and 80-year-old Swedish individuals. Community Dent Oral Epidemiol 25(2): 137-142.

21. Gilbert GH, Miller MK, Duncan RP, Ringelberg ML, Dolan TA, et al. (1999) Tooth-specific and person-level predictors of 24-month tooth loss among older adults. Community Dent Oral Epidemiol 27(5): 372-385.

22. Batista MJ, Lawrence HP, Maria da Luz Rosário de Sousa (2014) Impact of tooth loss related to number and position on oral health quality of life among adults. Health Qual Life Outcomes 12: 165.

23. Makhija SK, Gilbert GH, Boykin MJ, Litaker MS, Allman RM, et al. (2006) The relationship between sociodemographic factors and oral healthrelated quality of life in dentate and edentulous community-dwelling older adults. J Am Geriatr Soc 54(11): 1701-1712. 